\title{
EDITORIAL
}

\section{A PRÁTICA PROFISSIONAL DE ENFERMAGEM NOS ASPECTOS SOCIAISE POLÍTICOS}

\begin{abstract}
A organização social e política da profissão de Enfermagem tem sido objeto de grandes reflexões para a categoria profissional. 0 significativo número de profissionais, assim como os seus diferentes níveis de formação científico-tecnológico e sua atuação no sistema de saúde, pode ser encarado como vantagem e também como desvantagem, pois nossa identidade e visibilidade ainda carecem de estudos e intervenções na realidade de sua concretude. A própria corporação de Enfermagem não entende sua diversidade e seus números como um algo que lhe garanta poder, talvez pelo reconhecimento de seu déficit qualitativo de atuação, embora existam grupos e profissionais de destaque em suas determinadas áreas. M uitas vezes nosso ol har beira a endogenia, mas é preciso um ol har atento para o espaço social e político de nossa prática para que avancemos como profissão consolidada, de significativo interesse social, de força e de representação em todos os níveis da sociedade. N ossa prática profissional precisa de um plano nacional de mobilização de toda a categoria para realmente estar inserida nas questões sociais e políticas de nosso país, fazendo frente às demandas que a sociedade rei vindica nas questões de saúde. 0 cuidado, nossa prática genética, perpassa toda a dimensão sóciopolítica e demanda que, acima de quaisquer interesses e momentos pelos quais perpassam nossa categoria profissional e a sociedade, seja nossa bandeira primeira e seja nossa força de organização e atuação. É preciso que cada um de nós - enfermeiros, docentes, gerentes, pesquisadores, auxiliares e técnicos de enfermagem - faça uma enfermagem singular e que nossos espaços de atuação sejam marcados pelo toque de nossa presença enquanto profissionais políticos e socialmente engajados. As possibilidades de realizar cuidados diferenciados considerando redes sociais das comunidades e as políticas do país, bem como a sustentação dos trabalhos do enfermeiro e da equipe de enfermagem em fundamentos teóricos metodológicos provocarão visibilidade e confirmação da identidade profissional da enfermagem e, conseqüentemente, sua prática se fortalecerá nos aspectos políticos e sociais.
\end{abstract}

Prof. ${ }^{a}$ Dr. ${ }^{a}$ M aria Ribeiro Lacerda Chefe do Departamento de Enfermagem da Universidade Federal do Paraná - UFPR. Coordenadora do Núcleo de Ensino, Pesquisa e Extensão em Cuidado Humano de Enfermagem - NEPECHE 


\section{Social and political aspects in nursing professional practice}

Nursing social and political organization has been a major thought-provoking issue for the professional category. The meaningful number of its professionals, their different scientific and technological qualification levels as well as their performance in the heal th system can be viewed contrastively as positive and negative aspects provided that their identity and visibility still lack further studies and interventions in their reality. Even nursing caregivers do not realize their diversity and figures as a powerful instrument, maybe for acknowledging their qualitative performance deficit although there are outstanding teams and professionals in certain areas. Our look often gets to the verge of endogeny, however it is deemed necessary a careful look at the political and social realms of our practice so that we can get along as a consolidated profession of meaningful social concern, powerful and representative throughout all levels of society. O ur professional practice deems necessary a national mobilization plan for all the category in order to be effectively engaged with all political and social issues in our country, meeting society demands in heal th matters. Caring, our genetic practice, pervades all social and political dimensions and must be our flagship and drive for our organization and acting regardless any concerns and situations which our professional field and society have been going through. It makes necessary that all of us - nurses, nursing teachers, managers, researchers, nursing practitioners and assistants - perform our job in a unique way and that our working settings be marked by our presence while politically and socially engaged professionals. The possibilities to deliver diversified care considering community netw orks and the country policies, as well as nurses and nursing teams' work grounded by theoretical methodological bases will bring about visibility and ratification of their professional nursing identity; consequently, their practice will be strengthened in political and social aspects.

\section{La práctica profesional de enfermería en los aspectos sociales y políticos}

La organización social y política de la profesión de Enfermería viene siendo objeto de grandes reflexiones para esa categoría profesional. Podemos encarar el significativo número de profesionales, así como sus distintos niveles de formación científico y tecnológico y su acción en el sistema de salud como ventajas y también como desventajas, pues nuestra identidad y visibilidad todavía carecen de estudios e intervenciones en la realidad de su materialidad. La propia corporación de Enfermería no entiende su diversidad y sus números como al go que va a garantizar poder, quizá por el reconocimiento de su déficit cualitativo de actuación, a pesar de que existan grupos y profesionales de destaque en sus determinadas esferas de acción. M uchas veces, nuestra mirada se queda cerca de lo que está dentro, de lo que es sólo interno, pero es necesaria una mirada atenta para el espacio social y político de nuestra práctica, para que nosotros avancemos como profesión consolidada, de significativo interés social, de fuerza y de representación en todos niveles de la sociedad. Nuestra práctica profesional necesita de un plan nacional de movilización de toda la categoría a fin de realmente estar introducida en las cuestiones sociales y políticas de nuestro país, contestando a las demandas que la sociedad reivindica en las cuestiones de salud. El cuidado, nuestra práctica genética, pasa por medio de toda dimensión social y política y demanda que, además de cual esquier intereses y momentos por los cual es pasen nuestra categoría profesional y la sociedad, sea nuestra bandera primera e sea nuestra fuerza de organización y actuación. Es necesario que cada uno de nosotros - enfermeros, docentes, gerentes, investigadores, auxiliares y técnicos de enfermería - haga una enfermería singular y que nuestros espacios de actuación sean señalados por nuestra presencia mientras profesionales que participan de la política y de la sociedad. L as posibilidades de realizar cuidados diferenciados considerando redes sociales de las comunidades y las políticas del país, así como la sustentación de los trabajos del enfermero y del equipo de enfermería en fundamentos teóricos y metodológicos, causarán visibilidad y confirmación de la identidad profesional de la enfermería y, en consecuencia, su práctica se fortalecerá en los aspectos políticos y sociales.

Cogitare Enferm 2006 jan/abr; 11(1):7-8 